\title{
Negation as a means for emotion regulation? Startle reflex modulation during processing of negated emotional words
}

\author{
Cornelia Herbert • Roland Deutsch • Stefan Sütterlin • \\ Andrea Kübler • Paul Pauli
}

Published online: 3 March 2011

(C) Psychonomic Society, Inc. 2011

\begin{abstract}
This study investigated startle reflex modulation in 33 healthy student participants during the processing of negated emotional items. To build upon previous research, our particular interest was to find out whether processing of negated emotional items modulates emotional responding in line with the logical meaning of the negated expression, or instead leads to paradox emotional effects that point in the direction opposite the one logically implied by the negation. Startle reflex modulation was assessed during silent reading of pleasant and unpleasant nouns. The nouns were either paired with the possessive pronoun $m y$ or with the negation word no. The startle eyeblink amplitude was enhanced during processing of the unpleasant pronounnoun phrases and attenuated during processing of the pleasant phrases. Negation attenuated the startle eyeblink for negated unpleasant nouns and enhanced it for negated pleasant nouns. In line with this finding, negation decreased arousal ratings for unpleasant nouns and reversed the valence ratings for pleasant nouns. Our results are the first to show an effect of negation on both peripheral physiological and subjective indices of affective responding. Our results suggest that negation may be an effective strategy
\end{abstract}

C. Herbert $(\bowtie) \cdot$ S. Sütterlin · A. Kübler $\cdot$ P. Pauli Department of Psychology, University of Würzburg, Marcusstr. 9-11, 97070 Würzburg, Germany e-mail: cornelia.herbert@psychologie.uni-wuerzburg.de

R. Deutsch

Department of Psychology, Technische Universität Dresden, Zellescher Weg 17, 01069 Dresden, Germany

e-mail: deutsch@pychologie.tu-dresden.de

S. Sütterlin

INSIDE Research Centre, Université du Luxembourg,

Walferdange, Luxembourg for spontaneous down-regulation of emotional responses to unpleasant, but not to pleasant, stimuli.

Keywords Emotion · Negation · Startle reflex · Emotion regulation

\section{Introduction}

In everyday life, people often attempt to down-regulate their emotional responses to emotionally challenging stimuli by negating the stimulus' emotional implications. For example, people may try to calm themselves by thinking "this is not dangerous." Moreover, people often express their emotions by saying that they are not sad, anxious, or afraid, rather than by claiming the opposite. Two-step models of negation (e.g., Kaup, Zwaan, \& Lüdtke, 2007) have suggested that processing of negations relies on two mental simulations: a simulation of the negated state of affairs, and a simulation of the actual state of affairs. From this perspective, when applying negations to emotional expressions, people would first evaluate the content of the negated expression - for instance, fear - and then, in a subsequent step, evaluate the meaning of the negation-for instance, no fear. What are the emotional consequences of such activities?

On one hand, emotion negation could have paradox effects that resemble those found during emotion suppression. Instructing individuals to suppress their emotional reactions by telling them not to show any emotional reactions often results in an increase in physiological responding (Goldin, McRae, Ramel, \& Gross, 2008; Gross, 2002; Wegner, Shortt, Blake, \& Page, 1990), reduced positive affect (Gross \& Levenson, 1993, 1997; Nezlek \& Kuppens, 2008), and memory retrieval (Dillon, Ritchey, 
Johnson, \& LaBar, 2007; Richards \& Gross, 2000). Emotion negation could result in similar paradoxical effects, because the cognitive focus may still rest on the negated concept. Processing of such statements as it is not dangerous may activate danger and associated contents in memory (e.g., Giora, Balaban, Fein, \& Alkabets 2005; Hasson \& Glucksberg, 2006), and thereby maintain or even increase a state of avoidance motivation.

Some studies have already investigated the effects of negation on brief evaluative responses, often revealing a pattern resembling suppression (e.g., Deutsch, Gawronski, \& Strack, 2006; Draine, 1997). Drawing on evaluative priming, these studies have suggested that negated emotional prime words (e.g., no fear) have the same immediate affective priming effects as their affirmed counterparts (e.g., fear). Psycholinguistic studies based on semantic priming have reported similar effects (e.g., Giora et al., 2005; Hasson \& Glucksberg, 2006). Immediately after reading sentences containing negations, lexical decision studies have revealed semantic activation pointing in the direction opposite the one logically implied by the negation.

On the other hand, recent evidence on automatic emotion regulation has opened up the possibility that negation may be quite effective in modulating affective responses (Mauss, Evers, Wilhelm, \& Gross, 2006; Williams, Bargh, Nocera, \& Gray, 2009). Mauss et al. (2006) and Williams et al. (2009) observed that merely perceiving specific cue words modulates emotion processing in a manner that is similar to intentional emotion regulation. Perceiving words that signal emotional control (e.g., stable, restrain) resulted in reduced emotional responding in a subsequent emotion provocation task, relative to participants who perceived words related to strong affect (e.g., volatile, boiled). In addition, merely perceiving control cues was similarly effective in decreasing emotional responses to negative events, as if participants had been instructed to down-regulate their emotions intentionally by reappraising the situation. These findings suggest that stimuli associated with emotion regulation may trigger such regulation even in the absence of intentions to do so.

Given that people often deliberately use negation as a means of emotion regulation, the question arises of why emotion negation has appeared to be ineffective, or has even resulted in paradox effects, in previous studies using negation in paradigms that mapped automatic responses to stimuli. One reason may lie in the nature of the evaluative priming paradigms used in the previous research. Affective priming with evaluative decisions is largely based on mechanisms of response interference (Gawronski, Deutsch, LeBel, \& Peters, 2008), which are not specific to affect or emotion per se (Deutsch \& Gawronski, 2009; Klauer \& Musch, 2002). Perhaps, then, the paradox effects from negated affective stimuli observed in previous studies reflect specificities of response interference rather than of affective responding per se. In line with this suspicion, a recent experiment by Deutsch, Kordts-Freudinger, Gawronski, and Strack (2009) suggested that negations may have a strong impact on evaluative priming if the priming paradigm is not based on response interference (i.e., the affect misattribution procedure; Payne, Cheng, Govorun, \& Stewart, 2005). Moreover, previous studies of negation and affect severely limited the time available for processing the negated expressions. Extracting the meaning of negated concepts may however require considerable processing time (Hasson \& Glucksberg, 2006; Lüdtke, Friedrich, De Filippis, \& Kaup, 2008). As a consequence, the previous studies may have only allowed for limited inferences as to how negation shapes and regulates emotion processing in situations where people have more than a few hundred milliseconds to process the negated concepts.

The present research uses a paradigm with clearer emotional relevance than either affective or semantic priming. Also, we allowed for negation processing times well above the limit for successful negation processing as identified with multiword sentences (Hasson \& Glucksberg, 2006, Lüdtke et al., 2008). We assessed startle reflex modulation in healthy student volunteers who were instructed to process personally relevant unpleasant and pleasant nouns that were presented either unnegated (e.g., my fear, my success) or negated (e.g., no fear, no success). The words were presented for $4 \mathrm{~s}$, and startle tones were delivered not earlier than $1,800 \mathrm{~ms}$ after word onset. The acoustically elicited startle reflex is one of the most extensively used measures to assess participants' appetitive and defensive response tendencies elicited during spontaneous processing of emotional stimuli. Thus, as a measure of affective responding it appears to be most suitable for the online investigation of negation effects without confounding them with response interference effects. Startle modulation by emotional stimuli is a robust phenomenon that has been validated for picture stimuli (e.g., Bradley, Codispoti, Cuthbert, \& Lang, 2001; Bradley, Codispoti, \& Lang, 2006; Pauli, Diedrich, \& Müller, 2002), scenes (e.g., Jansen \& Frijda, 1994), sounds (Bradley \& Lang, 2000a), or word materials (e.g., Aitken, Siddle, \& Lipp 1999; Hazlett, Speiser, Goodman, Roy, Carrizal, Wynn et al., 2007; Herbert \& Kissler, 2010). In particular, these studies demonstrated that the acoustically elicited startle eyeblink response is potentiated during processing of unpleasant stimuli and inhibited during processing of pleasant stimuli, relative to processing of neutral stimuli as a baseline condition. In addition, startle modulation by emotional stimuli has been shown to be sensitive to changes in both perceived stimulus valence and emotional arousal (for an overview, see Bradley \& Lang, 2000b).

To summarize, we aimed to test the following hypotheses: If processing of negated emotional items modulates emo- 
tional responding in line with the logical meaning of the negated expression, the startle reflex should be potentiated during processing of negated pleasant expressions and inhibited during processing of negated unpleasant expressions, relative to the processing of unnegated, personally relevant emotional items. If, on the other hand, processing of negated items produces paradox effects and is thus inefficient in down-regulation of emotional responses, startle eyeblink modulation by negated and unnegated emotional expressions should not differ. Moreover, if the effects of negation are primarily arousal driven, startle modulation by negated unpleasant and pleasant verbal expressions should vary as a function of the words' perceived arousal values, as assessed by participants' rating data. Besides startle and individual rating data, we also assessed participants' memory performance in a behavioral free recall test to examine whether negation decreases the accessibility of emotional concepts stored in the mental lexicon.

\section{Method}

\section{Participants}

Thirty-three students of the University of Würzburg (12 male, 21 female; mean age 25 years), who were all righthanded native speakers of German, participated in the study. Participants reported normal audition and normal or corrected-to-normal vision, and none of them reported drug abuse, chronic somatic, neurological, or psychiatric diseases, or medication for any of these conditions. Participants were paid $€ 7$ in return for participation, and all of them gave written informed consent prior to the experiment.

\section{Stimuli}

The experimental stimuli comprised 36 unpleasant and 36 pleasant nouns, previously rated by 45 healthy volunteers for perceived valence and arousal using the SelfAssessment Manikin (SAM; Bradley \& Lang, 1994). Unpleasant and pleasant nouns did not differ significantly in mean arousal, but did differ in terms of stimulus valence. Additionally, unpleasant and pleasant nouns did not differ in concreteness, word length, or word frequency (see Table 1). The unpleasant nouns included words inducing negative emotions - in particular, words associated with threat (e.g., panic, fear). The pleasant nouns included words inducing positive emotions, particularly happiness (e.g., love, joy). The unpleasant and pleasant nouns were presented in two blocks. In each block, half of the nouns were presented in a negated fashion using the pronominal phrase no (e.g., no panic, no luck). The other half of the nouns were presented with the possessive pronoun $m y$ (e.g.,
Table 1 Stimulus material characteristics: Mean valence, arousal, and concreteness scores, as well as word length (number of letters) and word frequency counts (words per million), of the pleasant and unpleasant nouns used in the present study

\begin{tabular}{lll}
\hline & Pleasant & Unpleasant \\
\hline Valence & $7.40(0.11)$ & $2.55(0.11)$ \\
Arousal & $5.01(0.16)$ & $5.03(0.17)$ \\
Concreteness & $4.28(0.15)$ & $4.66(0.19)$ \\
Word length & $5.80(0.31)$ & $6.14(0.33)$ \\
Word frequency & $184.91(32.7)$ & $141.77(28.4)$ \\
\hline
\end{tabular}

Ratings range from 1 (extremely negative valence, extremely low arousal or concreteness) to 9 (extremely positive valence, extremely high arousal or concreteness). Standard errors are in parentheses. The word frequency counts for written language are based on the standardized CELEX Lexical Database (Baayen, Piepenbrock, \& Gulikers, 1995)

my fear, my success) to increase the emotional relevance of the stimuli and to induce strong and reliable emotional reactions. For each block, 36 additional, neutral stimuli consisting of meaningless letter strings served as baseline stimuli. In each block, unpleasant and pleasant words were randomly assigned to the two different stimulus presentation conditions (negated vs. unnegated), such that across blocks different sets of words were presented either negated or unnegated. Unpleasant, pleasant, and neutral stimuli were presented randomly in each block. The blocks were separated by an interval of $3 \mathrm{~min}$, and block order was randomized across participants.

Stimuli were presented in black letters (Times font, size $=$ 40) centered on a white background on a 15 -in. computer screen placed $80 \mathrm{~cm}$ away from the participants' eyes. Each stimulus was presented for $4 \mathrm{~s}$ and followed by an interstimulus interval with a variable duration of 1,800 2,200 ms. Startle tones were presented acoustically in both blocks on half of the trials, via stereo headphones during a time window of 1.8-3.5 s after stimulus onset. They consisted of $100-\mathrm{dB}$ sound pressure level bursts of white noise with a 50-ms duration and instantaneous rise and fall times.

Experimental runs were controlled by Presentation software (Neurobehavioral Systems. Inc., Albany, CA).

\section{Procedure}

Participants were familiarized with the laboratory settings, and the experiment was explained to them in general terms. They were then questioned about their handedness, health, and current mood state, and anxiety and depression scores were obtained via the PANAS scales (Watson, Clark, \& Tellegen, 1988), the State-Trait Anxiety Inventory (Laux, Glanzmann, Schaffner, \& Spielberger, 1981), and the Beck Depression Inventory (Hautzinger, Bailer, Worall, \& Keller, 1994). The participants did not differ in their self-report 
measures. All showed low depression scores $(M=6.09$, $S D=5.44)$ and normal anxiety scores, on both the trait $(M=43.3, S D=3.01)$ and state $(M=40.2, S D=5.09)$ levels, and reported more positive $(M=2.84, S D=0.55)$ than negative $(M=1.18, S D=0.30)$ affect.

Participants were seated in an electrically shielded, dimly lit, sound attenuated room. Electrodes for electromyographic recordings of the startle eyeblink response were attached, and the participants were given detailed instructions. They were told that they would be shown a series of personally relevant verbal expressions that would signal either the presence or the absence of an emotionally evocative experience. They were also told that during some trials, including baseline trials, a startling tone would be presented via headphones and that their task would be to read the words silently, to attend them and the neutral baseline stimuli, and to ignore the randomly occurring tones. After completion of the second block, participants performed a surprise free recall task, in which they were asked to remember as many of the verbal expressions presented in the second block as they could. Thereafter, they rated all stimuli for subjective valence and arousal using a 9-point paper-and-pencil version of the SAM (Bradley \& Lang, 1994) and were debriefed about the purpose of the experiment.

Physiological data collection and reduction

Electromyographic (EMG) recording: Startle eyeblink response The startle eyeblink response was measured electromyographically. Miniature $\mathrm{Ag} / \mathrm{Ag}$ electrodes $(5 \mathrm{~mm}$ in diameter) were attached at the left and right orbicularis oculi muscles beneath the left and right eyes. At both eyes, the interelectrode distance was $1 \mathrm{~cm}$. Recording and analysis of the startle data were performed according to the standard guidelines for startle eyeblink measurement (Blumenthal et al., 2005). EMG signals were recorded continuously with DC bandpass to $500 \mathrm{~Hz}$ and amplified by $500 \mathrm{~Hz}$ to be sampled at $1000 \mathrm{~Hz}$. The EMG electrodes were connected to ground and referenced to a skin electrode. Impedance was kept below $5 \mathrm{k} \Omega$ for all electrodes. Electromyographic activity was recorded with a V-Amp amplifier and analyzed with BrainVision Analyzer software (BrainProducts GmBH, Gilching, Germany). Offline, the raw EMG signal was filtered from $28-500 \mathrm{~Hz}$, rectified, and baseline corrected using the 100-ms interstimulus interval before onset of a trial. For each startle stimulus, startle peak amplitude and onset latency were scored and analyzed from the filtered, rectified, and baseline-corrected EMG signal. The exclusion criteria for baseline-corrected peak and latency scoring were a response onset before $20 \mathrm{~ms}$ or a response peak later than $200 \mathrm{~ms}$ after tone onset, as well as an onset-to-peak latency greater than $120 \mathrm{~ms}$. EMG peak amplitude (in microvolts) and peak latency (in milliseconds) were computed for each trial and averaged separately for each stimulus category, with trials with no detectable response scored as zero. On average, $4.5 \%$ of all trials were scored as zero responses, and $12.8 \%$ had to be excluded from further analysis because they did not accord with the above-defined exclusion criteria. The number of zero responses and discarded trials did not differ considerably between the different stimulation conditions.

\section{Statistical data analysis}

Startle eyeblink Modulation of the startle eyeblink amplitude and latency was analyzed with repeated measures ANOVAs including the factors Condition (negated pleasant, negated unpleasant, pleasant, unpleasant, and neutral), Eye (left and right), and Block (first and second).

Subjective ratings and free recall Participants' valence and arousal ratings, as well as their free recall memory performance for the correctly remembered stimuli, were analyzed with repeated measures ANOVAs, each of which included the factor Condition (negated pleasant, negated unpleasant, pleasant, and unpleasant).

For all data, significant ANOVA effects containing more than one degree of freedom in the numerator are reported after adjustment for violations of the sphericity assumption using the Greenhouse-Geisser procedure. Significant main effects and interactions were decomposed using planned comparison tests and Bonferroni-corrected $p$ values.

\section{Results}

\section{Startle eyeblink}

The startle eyeblink amplitude showed a significant main effect of condition $\left[F(4,128)=7.7(\varepsilon=.36), p<.01, \eta_{\mathrm{p}}{ }^{2}=.2\right]$. It was significantly larger during processing of unpleasant pronoun-noun phrases than during processing of pleasant pronoun-noun phrases or neutral baseline stimuli [unpleasant - pleasant, $F(1,32)=8.43, p<.01$; unpleasant - neutral, $F(1,32)=6.22, p<.05]$. During processing of pleasant pronoun-noun pairs, startle eyeblink amplitude was significantly inhibited relative to during processing of unpleasant pronoun-noun pairs, and reduced relative to startle amplitudes during processing of neutral baseline stimuli, although the pleasant-neutral difference was not significant [pleasant neutral, $F(1,32)=3.42, p=.07]$.

During processing of negated expressions, the startle eyeblink amplitude was enhanced by negated pleasant 
nouns, in comparison to both negated unpleasant nouns and neutral baseline stimuli, and was attenuated by negated unpleasant nouns, in comparison to negated pleasant nouns [negated pleasant - neutral, $F(1,32)=8.83, p<.01$; negated unpleasant - negated pleasant, $F(1,32)=8.83, p<.01]$. Startle amplitude modulation did not differ significantly for negated unpleasant nouns and neutral baseline stimuli [negated unpleasant - neutral, $F(1,32)=3.30, p>.1$ ]. However, it was significantly reduced during processing of negated unpleasant nouns versus unnegated unpleasant pronoun-noun pairs, and it increased during processing of negated pleasant nouns versus unnegated pleasant pronoun noun pairs [negated unpleasant - unpleasant, $F(1,32)=5.79$, $p<.05$; negated pleasant - pleasant, $F(1,32)=11.7, p<.01]$.

The main effects of eye $\left[F(1,32)=2.18, p>1.5, \eta_{\mathrm{p}}{ }^{2}=.06\right]$ and block $\left[F(1,32)=0.007, p>.9, \eta_{\mathrm{p}}{ }^{2}=.0002\right]$ were not significant. Latencies of the startle eyeblink amplitude showed no significant main effects of eye, condition, or block, nor any significant interactions of these variables (all $p \mathrm{~s}>.1$ ). The results are shown in Fig. 1 .

\section{Subjective ratings}

Unpleasant pronoun-noun pairs were judged as highly unpleasant, pleasant pronoun-noun pairs were judged as highly pleasant, and both were rated as similarly arousing by the participants (see Table 2). Negated unpleasant nouns were rated as less unpleasant and also as significantly less arousing than unnegated unpleasant pronoun-noun pairs [valence, $F(1,32)=55.4, p<.01$; arousal, $F(1,32)=29.9$, $p<.01]$. Negated pleasant nouns, by contrast, were judged as extremely unpleasant but as similar in arousal to unnegated unpleasant and pleasant pronoun-noun pairs (negated pleasant - pleasant: valence, $F(1,32)=152.0$, $p<.001$ ]. Participants' rating data are summarized in Table 2.

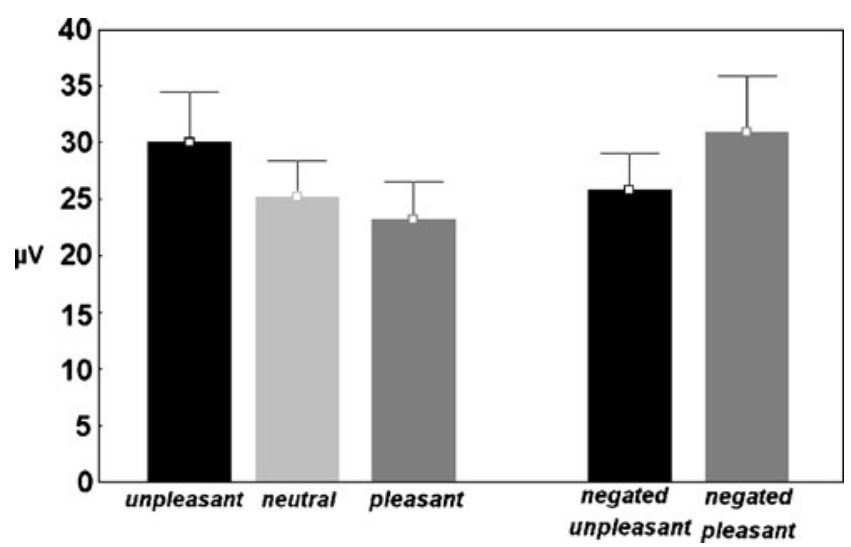

Fig. 1 Startle reflex modulation patterns: Modulation of the acoustically elicited EMG startle eyeblink response (means and standard errors) during processing of negated and unnegated pleasant and unpleasant nouns and of neutral, baseline stimuli
Table 2 Rating data: Participants' mean valence and arousal ratings for unnegated and negated pleasant and unpleasant nouns

\begin{tabular}{lll}
\hline & Pleasant & Unpleasant \\
\hline Valence & $7.22(0.29)$ & $2.86(0.27)$ \\
Arousal & $5.62(0.27)$ & $5.58(0.26)$ \\
& Negated Pleasant & Negated Unpleasant \\
Valence & $2.82(0.21)$ & $5.98(0.34)$ \\
Arousal & $5.77(0.22)$ & $3.32(0.35)$ \\
\hline
\end{tabular}

Ratings range from 1 (extremely negative valence, extremely low arousal) to 9 (extremely positive valence, extremely high arousal). Standard errors are in parentheses

\section{Memory: Free recall}

Free recall memory performance for correctly remembered nouns showed a significant main effect of condition $\left[F(3,96)=52.5(\varepsilon=.77), p<.01, \eta_{\mathrm{p}}{ }^{2}=.62\right]$. Pleasant, and particularly unpleasant, nouns were remembered worse when their meanings were negated [negated pleasant pleasant, $F(1,32)=49.5, p<.01$; negated unpleasant unpleasant, $F(1,32)=102.5, p<.01]$. The effects of negation on memory are presented in Fig. 2.

\section{Discussion}

The present study investigated whether the processing of negated and unnegated emotional items leads to spontaneous up- and down-regulatory effects on emotional responding. Extending previous research, we used startle reflex modulation as a paradigm to assess the emotional consequences of

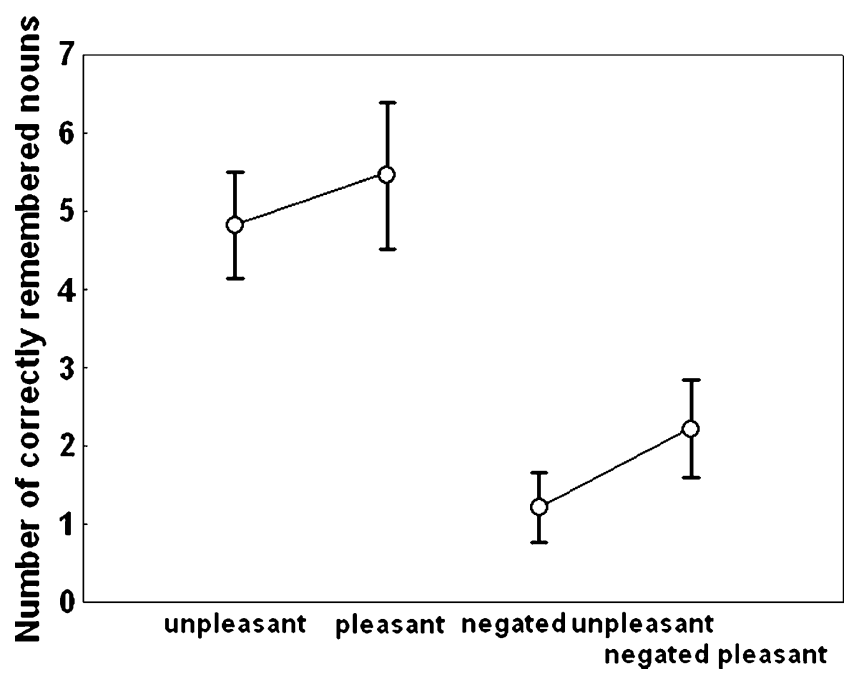

Fig. 2 Free recall memory performance: Memory for correctly remembered expressions, as obtained from the postexperimental free recall test 
spontaneous stimulus processing. Although participants were not instructed to regulate their emotional responses, they were given enough time to extract the full meaning of the negated items. To build upon previous results, we were particularly interested in whether, under such conditions, processing of negation cues modulates emotional responding in line with the logical meaning of the negated expression, or instead leads to paradox effects (i.e., effects pointing in the direction opposite the one logically implied by the negation). Negation had an impact on all measures of emotional responding, including modulation of startle eyeblink amplitude, memory, and subjective measures of perceived valence and arousal, all of which clearly differed from the effects observed during processing of unnegated emotional items.

Startle modulation during processing of unnegated emotional items

Startle eyeblink amplitude was enhanced during processing of words describing personally relevant unpleasant emotional experiences (i.e., unpleasant pronoun-noun pairs) and attenuated during processing of words describing personally relevant pleasant experiences (i.e., unpleasant pronoun-noun pairs). Theoretically, this emotional-valencedriven response pattern of the startle reflex is thought to reflect activation of organism's motivational systems of approach or avoidance (Lang, 1995). Empirically, it has been replicated many times during viewing of arousing unpleasant and pleasant pictures (Bradley et al., 2001; Bradley et al., 2006; Cuthbert, Schupp, Bradley, \& McManis, Lang, 1998; Pauli et al., 2002; Schupp, Cuthbert, Bradley, Birbaumer, \& Lang, 1997), although startle inhibition by arousing pleasant stimuli appears to be a less robust finding in the literature than startle potentiation by arousing unpleasant stimuli (e.g., Anokhin \& Golosheykin, 2010; Bradley et al., 2001). In particular, startle inhibition by arousing pleasant stimuli relative to neutral baseline conditions had previously been demonstrated only with erotic pictures (Bradley et al., 2001; Bradley et al., 2006). In the present study, the pleasant words were not exclusively of an erotic or sexual nature. Also, startle modulation by pleasant pronoun-noun pairs did not differ significantly from that by neutral baseline stimuli, but startle amplitudes were significantly inhibited during processing of pleasant pronoun-noun pairs relative to unpleasant pronoun-noun pairs. These results demonstrate that startle modulation by emotional stimuli, in line with the motivational priming hypothesis (Lang, 1995), also occurs with verbal, personally relevant emotional items, a finding that so far has remained challenged in the startle literature (e.g., Aitken et al., 1999; Herbert \& Kissler, 2010; Herbert, Kissler, Junghöfer, Peyk, \& Rockstroh, 2006).
Startle modulation during processing of negated emotional items

Startle modulation patterns elicited during processing of negated emotional items differed significantly from startle modulation patterns elicited during processing unnegated emotional items: Negating the meaning of unpleasant nouns attenuated the startle eyeblink potentiation observed during processing of unnegated unpleasant pronoun-noun pairs up to baseline level. Moreover, during processing of negated pleasant nouns, startle amplitudes were facilitated in a manner similar to the one found during processing of unpleasant pronoun-noun pairs (see Fig. 1). These startle modulation patterns were reliably observed for both left and right eyeblink responses and across stimulus presentation blocks. From the perspective of dual motivational systems we outlined above, our results suggest that negating decreases the response of the defensive system to unpleasant concepts but increases its response to pleasant concepts. In line with this finding, unpleasant nouns were judged as significantly less arousing and also as less unpleasant when negated. Negated pleasant nouns, on the other hand, were judged to be as highly arousing as unnegated pleasant or unpleasant pronoun-noun pairs. With respect to stimulus valence, participants evaluated negated pleasant nouns as being as unpleasant as unnegated unpleasant pronoun-noun pairs.

In addition, negated unpleasant and pleasant words were also remembered significantly worse than unnegated selfrelated emotional words during a postexperimental free recall test. ${ }^{1}$ This supports the view that the effects associated with emotion negation are driven by changes in both stimulus valence and arousal, and startle modulation during processing of negated pleasant and unpleasant nouns appears to interact with these changes.

Two explanations may account for these findings. First, it is possible that processing of negated concepts directly changes the valence of the stimulus. If this is the case, the expressions no fear and no luck, for instance, would immediately be appraised as positive and negative, respectively, and would determine which type of emotion (positive or negative) is elicited in the first place. In line with this suggestion, the effects of negation on emotional responding, as measured by startle reflex modulation in the present study, occurred quite spontaneously. Participants were not instructed to explicitly evaluate the negated expression according to specific criteria, nor were they asked to intentionally regulate their feelings in response to the presented stimuli. This evidence suggests that the

\footnotetext{
${ }^{1}$ One may argue, however, that this decrease in memory for negated emotional items in the present study was observed relative to selfrelated items, which have been shown to be better remembered, generally, than words without self-reference (for an overview, see Symons \& Johnson, 1997).
} 
processing of cue words can regulate emotion processing in a manner similar to the one shown during conditions of explicit, intentional emotion regulation (Mauss et al., 2006; Williams et al., 2009). Therefore, it is possible that in the present study, processing of the cue words $m y$ and no initiated fairly automatic regulation processes, which in turn may regulate activation in the motivational systems that prime approach and avoidance behavior, even without engendering intentional regulation processes. Such forms of spontaneous emotion regulation may constitute an important aspect of self-regulation reflecting fairly automatic and unintentional control of one's responses during exposure to emotionally evocative events (Bargh \& Williams, 2007).

The present observations are particularly interesting in light of theories of negation processing that have proposed that negation is not immediately incorporated into the evaluation process of the negated expression (e.g., Deutsch et al., 2006; Kaup et al., 2007). In line with this idea, previous studies have suggested that processing negated expressions, at least initially, activates the opposite of their logical meaning (Deutsch et al., 2006; Draine, 1997; Giora et al., 2005; Hasson \& Glucksberg, 2006). However, previous studies have also suggested that with processing times comparable to those in the present study, the logical meaning of the negated expressions can be incorporated in automatic activation processes (Hasson \& Glucksberg, 2006; Lüdtke et al., 2008). The present study extended this to the investigation of startle reflex modulation. Nevertheless, whether the effects of negation on emotional responding are driven by primary appraisal processes or, as suggested by two-step models, by reappraisal must be subjected to further investigation. To examine this question, future studies will be needed to investigate the effects of negation, in one experiment and one participant group, across the entire time line of affective responding.

In summary, our study has provided important insights into some of the boundary conditions supporting effects of negation in an emotional context. Extending previous research, our study demonstrated that with fairly long processing times and no explicit evaluation or regulation instructions, emotional responses such as the startle reflex are modulated in line with the logical meaning of the negated expression - that is, they are reduced during processing of negated unpleasant content and are enhanced during processing of negated pleasant content. Thus, negation may be an effective strategy for spontaneous down-regulation of emotional responses to unpleasant experiences, but not for responses to pleasant ones. Further investigation into the intricacies of negation will show how the effects of negation during spontaneous emotion regulation (present study) contrast with active emotion regulation conditions in which participants are explicitly instructed to up- or down-regulate their responses to emotional expressions by means of negation.
Assessments of additional outcome measures beyond startle (e.g., event-related brain potentials) and comparisons of effects across tasks (e.g., passive vs. active evaluation and regulation paradigms) would be especially fruitful to this end.

Acknowledgement We thank Anca Sfärlea and Thomas Kaufmayer for help with data collection. This research was supported by the German Research Foundation (DFG). We thank Kevin Ochsner and the anonymous referees for their helpful comments.

\section{References}

Aitken, C. J., Siddle, D. A., \& Lipp, O. V. (1999). The effects of threat and nonthreat word lead stimuli on blink modification. Psychophysiology, 36, 699-705.

Anokhin, A. P., \& Golosheykin, S. (2010). Startle modulation by affective faces. Biological Psychology, 83, 37-40.

Baayen, R. H., Piepenbrock, R., \& Gulikers, L. (1995). The CELEX Lexical Database (CD-ROM). Philadelphia, PA: Linguistic Data Consortium, University of Pennsylvania.

Bargh, J. A., \& Williams, L. E. (2007). The nonconscious regulation of emotion. In J. Gross (Ed.), Handbook of emotion regulation (pp. 429-445). New York: Guilford Press.

Blumenthal, T. D., Cuthbert, B. N., Filion, D. L., Hackley, S., Lipp, O. V., \& van Boxtel, A. (2005). Committee report: Guidelines for human startle eyeblink electromyographic studies. Psychophysiology, 42, $1-15$.

Bradley, M. M., Codispoti, M., Cuthbert, B. N., \& Lang, P. J. (2001). Emotion and motivation I: Defensive and appetitive reactions in picture processing. Emotion, 1, 276-298.

Bradley, M. M., Codispoti, M., \& Lang, P. J. (2006). A multi-process account of startle modulation during affective perception. Psychophysiology, 43, 486-497.

Bradley, M. M., \& Lang, P. J. (1994). Measuring emotion: The SelfAssessment Manikin and the semantic differential. Journal of Behavior Therapy and Experimental Psychiatry, 25, 49-59.

Bradley, M. M., \& Lang, P. J. (2000a). Affective reactions to acoustic stimuli. Psychophysiology, 37, 204-215.

Bradley, M. M., \& Lang, P. J. (2000b). Measuring emotion: Behavior, feeling and physiology. In R. Lane \& L. Nadel (Eds.), Cognitive neuroscience of emotion (pp. 242-276). New York: Oxford University Press.

Cuthbert, B. N., Schupp, H. T., Bradley, M., McManis, M., \& Lang, P. J. (1998). Probing affective pictures: Attended startle and tone probes. Psychophysiology, 35, 344-347.

Deutsch, R., \& Gawronski, B. (2009). When the method makes a difference: Antagonistic effects on "automatic evaluations" as a function of task characteristics of the measure. Journal of Experimental Social Psychology, 45, 101-114.

Deutsch, R., Gawronski, B., \& Strack, F. (2006). At the boundaries of automaticity: Negation as reflective operation. Journal of Personality and Social Psychology, 91, 385-405.

Deutsch, R., Kordts-Freudinger, R., Gawronski, B., \& Strack, F. (2009). Fast and fragile: A new look at the automaticity of negation processing. Experimental Psychology, 56, 434-446.

Dillon, D. G., Ritchey, M., Johnson, B. D., \& LaBar, K. S. (2007). Dissociable effects of conscious emotion regulation strategies on explicit and implicit memory. Emotion, 7, 354-365.

Draine, S. C. (1997). Analytic limitations of unconscious language processing. University of Washington, Seattle: Unpublished doctoral dissertation.

Gawronski, B., Deutsch, R., LeBel, E. P., \& Peters, K. R. (2008). Response interference as a mechanism underlying implicit 
measures: Some traps and gaps in the assessment of mental associations with experimental paradigms. European Journal of Psychological Assessment, 24, 218-225.

Giora, R., Balaban, N., Fein, O., \& Alkabets, I. (2005). Negation as positivity in disguise. In H. L. Colston \& A. Katz (Eds.), Figurative language comprehension: Social and cultural influences (pp. 233-258). Mahwah, NJ: Erlbaum.

Goldin, P. R., McRae, K., Ramel, W., \& Gross, J. J. (2008). The neural bases of emotion regulation: Reappraisal and suppression of negative emotion. Biological Psychiatry, 63, 577586.

Gross, J. J. (2002). Emotion regulation: Affective, cognitive, and social consequences. Psychophysiology, 39, 281-291.

Gross, J. J., \& Levenson, R. W. (1993). Emotional suppression: Physiology, self-report, and expressive behavior. Journal of Personality and Social Psychology, 64, 970-986.

Gross, J. J., \& Levenson, R. W. (1997). Hiding feelings: The acute effects of inhibiting negative and positive emotion. Journal of Abnormal Psychology, 106, 95-103.

Hasson, U., \& Glucksberg, S. (2006). Does understanding negation entail affirmation? An examination of negated metaphors. Journal of Pragmatics, 38, 1015-1032.

Hautzinger, M., Bailer, M., Worall, H., \& Keller, F. (1994). BeckDepressions-Inventar (BDI). Bern, Switzerland: Huber.

Hazlett, E. A., Speiser, L. J., Goodman, M., Roy, M., Carrizal, M., Wynn, J. K., et al. (2007). Exaggerated affect-modulated startle during unpleasant stimuli in borderline personality disorder. Biological Psychiatry, 62, 250-255.

Herbert, C., \& Kissler, J. (2010). Motivational priming and processing interrupt: Startle reflex modulation during shallow and deep processing of emotional words. International Journal of Psychophysiology, 76, 64-71.

Herbert, C., Kissler, J., Junghöfer, M., Peyk, P., \& Rockstroh, B. (2006). Processing of emotional adjectives: Evidence from startle EMG and ERPs. Psychophysiology, 43, 197-206.

Jansen, D. M., \& Frijda, N. H. (1994). Modulation of the acoustic startle response by film-induced fear and sexual arousal. Psychophysiology, 31, 565-571.

Kaup, B., Zwaan, R. A., \& Lüdtke, J. (2007). The experiential view of language comprehension: How is negation represented? In F. Schmalhofer \& C. A. Perfetti (Eds.), Higher level language processes in the brain: Inference and comprehension processes (pp. 255-288). Mahwah, NJ: Erlbaum.
Klauer, K. C., \& Musch, J. (2002). Goal-dependent and goalindependent effects of irrelevant evaluations. Personality and Social Psychology Bulletin, 28, 802-814.

Lang, P. J. (1995). The emotion probe. American Psychologist, 50, $372-385$

Laux, L., Glanzmann, P., Schaffner, P., \& Spielberger, C. D. (1981). Das State-Trait-Angstinventar (STAI). Weinheim, Germany: Beltz.

Lüdtke, J., Friedrich, C. K., De Filippis, M., \& Kaup, B. (2008). Event-related potential correlates of negation in a sentencepicture verification paradigm. Journal of Cognitive Neuroscience, 20, 1355-1370.

Mauss, I. B., Evers, C., Wilhelm, F. H., \& Gross, J. J. (2006). How to bite your tongue without blowing your top: Implicit evaluation of emotion regulation predicts affective responding to anger provocation. Personality and Social Psychology Bulletin, 32, $589-602$

Nezlek, J. B., \& Kuppens, P. (2008). Regulating positive and negative emotions in daily life. Journal of Personality, 76, 561-579. doi:10.1111/j.1467-6494.2008.00496.x

Pauli, P., Diedrich, O., \& Müller, A. (2002). Covariation bias in the affect-modulated startle paradigm. Journal of Behavior Therapy and Experimental Psychiatry, 33, 191-202.

Payne, B. K., Cheng, C. M., Govorun, O., \& Stewart, B. D. (2005). An inkblot for attitudes: Affect misattribution as implicit measurement. Journal of Personality and Social Psychology, 89, 277-293.

Richards, J. M., \& Gross, J. J. (2000). Emotion regulation and memory: The cognitive costs of keeping one's cool. Journal of Personality and Social Psychology, 79, 410-424.

Schupp, H. T., Cuthbert, B. N., Bradley, M. M., Birbaumer, N., \& Lang, P. J. (1997). Probe P3 and blinks: Two measures of affective startle modulation. Psychophysiology, 34, 1-6.

Symons, C. S., \& Johnson, B. T. (1997). The self-reference effect in memory: A meta-analysis. Psychological Bulletin, 121, 371-394.

Watson, D., Clark, L. A., \& Tellegen, A. (1988). Development and validation of brief measures of positive and negative affect: The PANAS scales. Journal of Personality and Social Psychology, 54, 1063-1070. doi:10.1037/0022-3514.54.6.1063

Wegner, D. M., Shortt, J. W., Blake, A. W., \& Page, M. S. (1990). The suppression of exciting thoughts. Journal of Personality and Social Psychology, 58, 409-418.

Williams, L. E., Bargh, J. A., Nocera, C. C., \& Gray, J. R. (2009). The unconscious regulation of emotion: Nonconscious reappraisal goals modulate emotional reactivity. Emotion, 9, 847-854. 